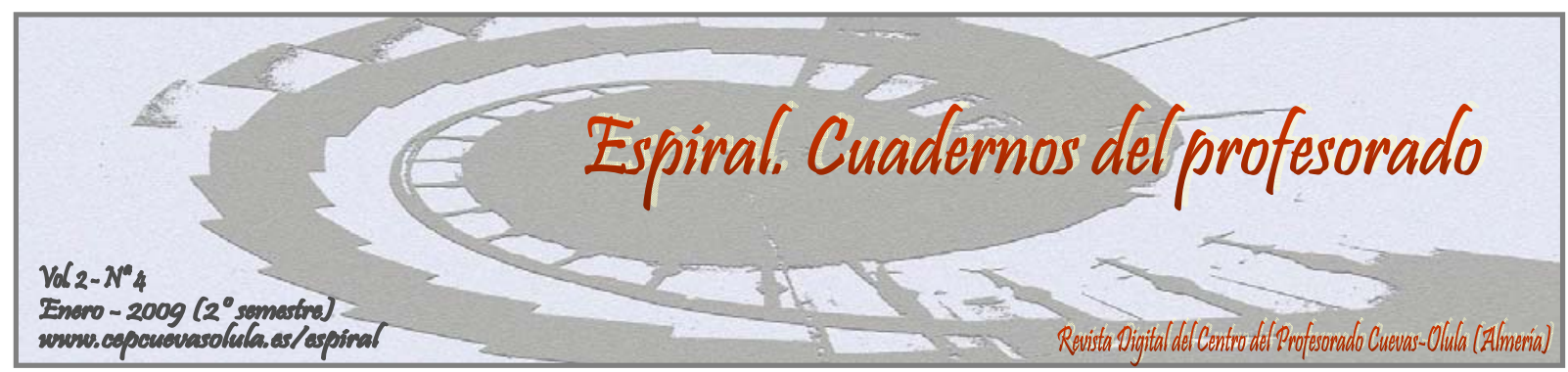

\title{
PROPUESTA DIDÁCTICA PARA FOMENTAR LA LECTURA Y EL RESPETO A LA DIVERSIDAD CULTURAL
}

\section{CLASSROOM ACTIVITIES TO FOSTER READING AND RESPECT TOWARDS CULTURAL DIVERSITY}

\section{Beatriz Martínez Serrano}

\section{Profesora de Lengua Castellana y Literatura en el IES Miguel Crespo, Fernán Núñez, Córdoba}

RESUMEN: A través de nuestra propuesta didáctica, diseñada para $1^{\circ}$ de ESO, aspiramos a suscitar el interés por la lectura como fuente de placer y vía de acceso al conocimiento y a la información, así como a potenciar el acercamiento a otras culturas ajenas a la propia. Del mismo modo, contribuiremos a la adquisición de las ocho competencias básicas mediante distintos tipos de cuestiones. Abogaremos por una metodología activa y participativa en la que el alumnado sea el verdadero protagonista del proceso de enseñanza-aprendizaje y nos decantaremos por un enfoque interdisciplinar. El libro de literatura juvenil titulado Laila, escrito por Laila Karrouch, se erigirá en nuestro punto de partida para alcanzar los objetivos previamente mencionados. Antes, durante y después de la lectura, los alumnos y alumnas tendrán que resolver una amplia gama de actividades que se articulan en torno a tres grandes bloques, subdivididos a su vez en diferentes apartados. Finalizadas las actividades propuestas, el alumnado descubrirá las maravillosas historias que el apasionante mundo de la literatura pone a nuestro alcance y valorará la riqueza que aporta la diversidad lingüística y cultural a nuestra sociedad.

Palabras clave: lectura, interculturalidad, respeto, competencias, interdisciplinariedad.

ABSTRACT: By means of this classroom activities, which is intended for $1^{\text {st }}$-year ESO students, we intend to foster students' interest in reading as an enjoyable activity and as a means of access to knowledge and information, as well as to bring about a sensible approach to cultures other than one's own. Similarly, we will contribute to the acquisition of the so-called basic competences by means of different activity types. We will try to employ an active methodology in which students are the true protagonists of the teaching-learning process and interdisciplinarity will be taken into account throughout. The teenagers' book entitled Laila, by Laila Karrouch, will be our starting point to achieve the above mentioned objectives. Students will be required to complete a number of before-, while- and post-reading activities, which are organized around three basic categories, subdivided into several parts. Once the activities are finished, students will find out more about the amazing stories that literature gives us and will acknowledge the importance of the linguistic and cultural diversity in our present world.

Key words: reading, interculturalism, respect, competences, interdisciplinarity.

Martínez Serrano, B. (2009). Propuesta didáctica para fomentar la lectura y el respeto a la diversidad cultural. Espiral. Cuadernos del Profesorado [en línea], 2(4), 48-55. Disponible en: http://www.cepcuevasolula.es/espiral. 


\section{1.- INTRODUCCIÓN.}

Dos de los grandes retos del profesorado radican en fomentar la lectura como fuente de placer y vía de acceso al conocimiento y a la información, por un lado, y, por otro lado, inculcar al alumnado una serie de valores positivos que le permitan desarrollarse como ciudadanos/as responsables en el seno de una sociedad caracterizada por la diversidad lingüística y cultural. La propuesta didáctica que ofrecemos, diseñada para $1^{\circ}$ de ESO, tiene como objetivo prioritario abordar ambas cuestiones, pues se nos antojan de vital importancia en el mundo de la docencia. No en vano el Real Decreto 1631/2006 de 29 de diciembre, por el que se establecen las enseñanzas mínimas correspondientes a la Educación Secundaria Obligatoria, concede un lugar privilegiado a la lectura al considerarla un factor clave para el desarrollo de las competencias básicas y al establecer un tiempo obligatorio para su práctica en todas las materias del currículo.

En lo que atañe a la educación en valores, conviene precisar que desempeña un papel crucial en la medida en que resulta imprescindible con vistas a lograr el primer objetivo recogido en el mencionado Real Decreto, a saber, el relativo a la asunción responsable de derechos en el respeto a los demás, la práctica de la tolerancia, la cooperación y la solidaridad entre las personas y grupos, el ejercicio del diálogo y el afianzamiento de los derechos humanos como valores comunes de una sociedad plural, y la preparación para la ciudadanía democrática. Dentro del marco de la educación en valores, centraremos nuestra atención en la interculturalidad. Partiendo de la comprensión de Andalucía como lugar de encuentro y convivencia de diversas culturas, fomentaremos el respeto hacia la multiplicidad cultural de nuestra Comunidad Autónoma.

Desde la publicación del Plan de Fomento de la Lectura, una de cuyas metas más ambiciosas era la de acercar el libro y la lectura a los ciudadanos y las ciudadanas con el fin de garantizar un acceso igualitario a los recursos culturales y de información, han sido muchas las voces que se han unido a la defensa a ultranza de propuestas que promuevan la lectura dentro y fuera de los centros educativos. En esta línea se sitúa la opinión de Castán, quien, a propósito del fomento de los hábitos lectores, sostiene que "resulta una actividad muy importante que no puede descuidar una escuela cuyo objetivo prioritario es, sin embargo, el desarrollo de la comprensión y de la habilidad lectora y el fomento de los hábitos de estudio y de trabajo intelectual" (2002, p. 148). No obstante, se trata de una tarea no exenta de dificultades, dado que, tal y como reconoce Martín, "es un proceso lento, lleno de altibajos, unas veces con sabor agrio y otras veces con momentos dulces, que debemos afrontar con decisión, paciencia, formación $y$, sobre todo, con mucho amor" (2005, p. 23). También Lage comparte esta opinión: "Formar un lector, educar su sensibilidad, crear el hábito de la lectura, es un trabajo en ocasiones complicado, [...] pero a la larga muy gratificante” (2006, p. 73).

De cualquier modo, al margen de los obstáculos, la escuela tiene un compromiso ineludible con la lectura, ya que numerosos estudios ponen de manifiesto que el aumento de capacidad para la misma deriva en un aumento de capacidad para el aprendizaje en general (Bamberger, 1975). En este sentido, "decir que un adolescente lee equivale a decir que se trata de un joven que obtiene mejores resultados académicos, que tiene menores dificultades para leer y que es más creativo e imaginativo" (Murillo et al., 2003, p. 178). A dicho respecto, cabe destacar que se ha demostrado empíricamente la importancia del fomento de los hábitos lectores para crear a ciudadanos/as más libres, inteligentes, sabios/as y creativos/as. Por tanto, coincidimos con Mata en que "una de las misiones más gratas de un profesor es la de convertirse en maestro de lectura y transformar las aulas en escuelas de lectura" (2004, p. 53). Ahora bien, llegados a este punto es preciso aclarar lo que entendemos por enseñar a leer: "es infundir en los alumnos el deseo de adentrarse en una dimensión desconocida de la experiencia humana, es arraigar la convicción de que existen otras vidas dignas de ser vividas, aun a través de la ficción" (Mata, 2004, p. 53).

El libro titulado Laila, escrito por Laila Karrouch, será el punto de partida para suscitar el interés por la lectura $\mathrm{y}$, al mismo tiempo, nos brindará la oportunidad de acercar a nuestros/as alumnos/as a otras culturas distintas a la propia. Dicho acercamiento será esencial a la hora de garantizar la convivencia pacífica y el respeto hacia la sociedad plurilingüe y pluricultural en que nos hallamos inmersos/as. De este modo, contribuiremos a la consecución de uno de los principales objetivos del II Plan Integral para la 
inmigración en Andalucía, el de potenciar la sensibilización social sobre los valores positivos de la inmigración, mediante el rechazo del racismo, la xenofobia y cualquier tipo de discriminación que se produzca. Asimismo, contribuiremos a la adquisición de las denominadas competencias básicas mediante cuestiones de diversa índole y adoptando un enfoque interdisciplinar. Nuestra propuesta didáctica se articula en torno a tres bloques fundamentales: actividades de iniciación a la lectura, actividades de profundización en la lectura y actividades de ampliación de la lectura. Antes de adentrarnos en el origen de la experiencia, esbozaremos el contenido de Laila.

Laila es la protagonista de la estremecedora historia a la que da nombre. Con tan sólo ocho años, se ve obligada a abandonar junto a su familia el país que la vio nacer, Marruecos, dado que deciden emigrar a España en busca de unas condiciones de vida más prósperas. Superadas las dificultades económicas y la barrera del idioma en un primer momento, la joven se adaptará perfectamente a las costumbres típicas de nuestro país, sin olvidar en ningún instante sus raíces ni su cultura. Ella misma reconoce que, lejos de perder su cultura y sus raíces, ha ganado otra cultura y otras costumbres. De hecho, el conocer dos culturas tan distintas y opuestas ha sido una experiencia muy gratificante y enriquecedora, al enseñarle a convivir con personas de mentalidades muy diferentes y a valorar más a la gente, compartir sentimientos e intercambiar opiniones.

\section{2.- ORIGEN DE LA EXPERIENCIA.}

Los resultados de las pruebas de evaluación inicial, realizadas en $1^{\circ}$ de ESO a comienzos de curso, ponen de manifiesto que buena parte del alumnado presenta problemas relacionados con la comprensión de textos de diversa índole, así como carencias y limitaciones a la hora de expresarse de forma adecuada. Este hecho, unido a la constatación de que la mayoría no ha adquirido el hábito de la lectura ni la ha incorporado a su vida cotidiana, nos lleva a plantearnos la necesidad de dedicar al menos una de las cuatro horas semanales de la materia de Lengua Castellana y Literatura al desarrollo exclusivo de prácticas de lectura obligatorias sobre un libro por trimestre, pues son múltiples las ventajas derivadas de la misma: potencia la imaginación, da a conocer nuevos mundos y experiencias, incrementa el vocabulario, permite interiorizar estructuras gramaticales de distinto grado de dificultad, favorece la correcta expresión tanto oral como escrita, abre nuestra mente, entre otras.

Sin lugar a dudas, la lectura, hábito que ha de estimularse mediante su práctica regular (Gardner y Myers, 2005), es uno de los ejercicios más completos y fructíferos, especialmente si va acompañada de una serie de actividades que susciten la reflexión, la creatividad y la indagación. Las razones aducidas son más que suficientes para demostrar la conveniencia de plantear las clases de Lengua Castellana y Literatura en torno a la lectura de textos variados y representativos de las distintas formas textuales y géneros literarios. No obstante, dicha medida no parece suficiente. Es altamente recomendable incluir una serie de lecturas obligatorias que el alumnado ha de realizar en casa, dado que se ha comprobado que cuanto mayor es el número de lecturas obligatorias que se imponen, más leen los alumnos y las alumnas (Murillo et al., 2003), y que "sólo leyendo se forman lectores" (Lage, 2006, p. 67). Asimismo, conviene consagrar un tiempo de las clases de Lengua y Literatura, "ámbito privilegiado para el fomento de la lectura" (Murillo et al., 2003, p. 171), a practicar la lectura, tanto silenciosa como en voz alta, de un libro acorde con la psicología de los/as adolescentes, y qué mejor espacio que la biblioteca escolar para acometer esta apasionante tarea. La elección de un buen libro entraña una gran dificultad, sobre todo si tenemos en cuenta que no siempre coinciden los gustos e intereses del profesorado con los del alumnado. Así pues, "lo que puede parecer bueno al maestro puede no parecérselo al alumno" (Casas y Centellas, 1989, p. 23). A menudo, los/as docentes intentamos conjugar dos aspectos cruciales: un libro que conecte con las inquietudes de nuestros/as alumnos/as y, al mismo tiempo, que nos permita abordar alguna cuestión de relevancia en la actualidad. Éste es el caso de la obra seleccionada, Laila, que, además de suscitar el interés de los/as lectores/as por estar narrada por una chica joven que relata su vida en primera persona, plasma el tema de la inmigración y la adaptación a una cultura nueva y completamente diferente sin perder de vista la propia.

\section{3.- OBJETIVOS.}

A grandes rasgos, los objetivos que se pretenden lograr son los que se especifican a continuación: 
$\square$ Inculcar el hábito de la lectura como fuente de placer y como vía de acceso al conocimiento.

$\square$ Fomentar el respeto hacia la diversidad lingüística y cultural que caracteriza a la sociedad de nuestros días.

$\square$ Contribuir a la adquisición de las ocho competencias básicas y, de un modo especial, de la competencia en comunicación lingüística y de la competencia social y ciudadana.

$\square$ Mejorar la comprensión y la expresión tanto oral como escrita del alumnado.

$\square$ Incrementar el acervo léxico de nuestros alumnos y nuestras alumnas.

$\square$ Potenciar el uso de las nuevas tecnologías de la información y la comunicación en el proceso de enseñanza-aprendizaje.

$\square$ Suscitar un pensamiento crítico y reflexivo ante información de naturaleza diversa.

$\square$ Tomar conciencia de la sociedad plurilingüe y pluricultural en que nos hallamos inmersos/as.

$\square$ Valorar la lengua como vehículo de expresión de las distintas culturas.

$\square$ Interesarse por el aprendizaje de idiomas en una sociedad cada vez más mundializada.

\section{4.- METODOLOGÍA.}

Nos decantamos por una metodología activa y participativa en la que el alumnado se convierte en el protagonista indiscutible del proceso de enseñanza-aprendizaje. El profesorado, por su parte, se erige en el guía que va orientándolo en ese camino que lo conducirá a la ampliación de conocimientos de diversa índole, a la adquisición de habilidades y al desarrollo de una serie de competencias básicas que le permitirán integrarse con éxito en la sociedad. A la hora de fomentar el aprendizaje por descubrimiento, resultan imprescindibles las nuevas tecnologías de la información y la comunicación, que ponen a nuestro servicio una fuente inagotable de recursos. Los alumnos y alumnas tendrán que ser capaces de acceder a la información y discernir los datos relevantes de aquellos otros que carecen de interés.

Junto al trabajo individual, potenciaremos el trabajo en equipo, puesto que lo consideramos muy fructífero y enriquecedor. De hecho, brinda la posibilidad de intercambiar opiniones, compartir puntos de vista diferentes, trabajar valores como el respeto y la solidaridad, y, en definitiva, enriquecerse mutuamente. Dentro de la amplia y variada gama de actividades que hemos diseñado, pese a que predominan las encaminadas a contribuir a la adquisición de la competencia en comunicación lingüística y la competencia social y ciudadana, hemos incluido cuestiones de carácter interdisciplinar, con el propósito de que el alumnado perciba que lo que aprende en una determinada materia puede extrapolarlo a otras. De esta manera, asimilará que el conocimiento no se puede dividir en compartimentos estancos, sino que todo se halla interrelacionado. Asimismo, conectaremos las actividades planteadas con la realidad más inmediata de nuestros/as alumnos/as, a fin de hacer explícito el carácter práctico y utilitario de lo que se aprende en las aulas. Esta metodología que hemos adoptado se encuentra en la misma línea que la contemplada en el Decreto 231/2007 de 31 de julio, por el que se establece la ordenación y las enseñanzas correspondientes a la Educación Secundaria Obligatoria en Andalucía: "La metodología didáctica en esta etapa educativa será fundamentalmente activa y participativa, favoreciendo el trabajo individual y cooperativo del alumnado en el aula e integrará en todas las materias referencias a la vida cotidiana y al entorno del alumnado".

\section{5.- CORPUS DE ACTIVIDADES.}

Tal y como hemos apuntado en la introducción, las actividades confeccionadas se hallan organizadas en torno a tres grandes grupos: actividades de iniciación a la lectura, actividades de profundización en la lectura y actividades de ampliación de la lectura. A su vez, el segundo y el tercer bloque presentan varios sub-apartados.

\section{I}

\section{Actividades de iniciación a la lectura}

1. ¿Cuáles son los tres grandes géneros literarios?

2. ¿A qué género pertenece el libro titulado Laila?

3. Lee la información que aparece al final de la historia sobre Laila Karrouch, autora de la misma, y recoge los datos más relevantes en forma de esquema. 
4. Busca en un diccionario (impreso o digital) el significado de la palabra interculturalidad.

\section{II}

\section{Actividades de profundización en la lectura}

\section{Contenido}

5. Resume con tus propias palabras el contenido del libro que acabas de leer.

6. Responde a las siguientes cuestiones:

- ¿Cuántos hermanos tenía Laila? Indica sus nombres.

- ¿QQué medios de transporte utiliza la familia para salir por primera vez de su país?

- ¿Qué nombre recibía su pueblo de origen?

- ¿Cuál es la religión que practican Laila y sus familiares?

- ¿Cuál era el nombre de la calle en la que vivían cuando se mudaron de país?

- ¿Dónde trabajaba su padre?

- ¿A qué familiares echa de menos la joven al llegar a su nuevo hogar?

- ¿ ¿A qué edad comenzaban el colegio en Marruecos?

- ¿Cuál fue la primera frase que el padre de Laila le enseñó en español?

- ¿Qué fiesta típica de la religión cristiana celebraron?

- ¿Qué deporte le encantaba practicar a Laila?

- ¿En qué tipo de competiciones participaba?

- ¿Qué medio de transporte empleaba el pescadero en Marruecos para trasladar su mercancía?

- ¿ ¿Qué matrimonio se dejó pactado durante las primeras vacaciones en Marruecos?

- ¿Qué enfermedad padecía la abuela de Holanda?

- ¿Con quién se comprometió Laila?

- ¿Dónde estaba trabajando cuando se quedó embarazada?

- ¿QQué estudios comenzó a realizar por su cuenta cuando ya estaba en estado de buena esperanza?

- ¿Cuál fue el nombre que eligió para su hija?
- ¿Qué sucede al final de la historia?

7. Según su argumento, ¿̇ante qué tipo de novela nos encontramos?: aventuras, humor, realidad social, misterio o ciencia ficción.

\section{Estructura}

8. ¿¿De cuántos capítulos consta el libro?

9. ¿Qué tres partes podemos diferenciar en todo texto narrativo? Explícalas brevemente.

10. ¿En qué persona están narrados los hechos?

11. ¿Quién es el narrador o narradora de la historia?

12. ¿De qué tipo de narrador/a se trata? Justifica tu respuesta.

\section{Personajes}

13. ¿Qué personajes aparecen en la historia? Distingue entre principales y secundarios y comenta las relaciones que existen entre ellos.

14. ¿Cuál era el nombre de la primera vecina que se despide de Laila cuando ésta se disponía a abandonar su pueblo natal?

15. ¿Cómo se llamaba su primera maestra en España? Indica los rasgos físicos y de su carácter que aparecen en la descripción que Laila hace de ella.

16. ¿Cómo te imaginas al personaje de Laila? Realiza un retrato de la misma. No olvides que has de incluir su descripción física y psicológica.

17. Dibuja a Laila atendiendo a la descripción física que has hecho de ella.

\section{Lugar}

18. ¿En qué país nació Laila y pasó los primeros años de su infancia?

19. ¿A qué país emigró con la intención de mejorar sus condiciones de vida?

20. ¿Cuál era el país de origen de la madre de Laila?

21. ¿En qué país europeo vivían sus abuelos maternos?

\section{Tiempo}

22. ¿Cuándo abandona Laila su pueblo?

23. ¿Cuánto tiempo tardaron en volver de vacaciones a Marruecos una vez que se establecieron en España?

24. ¿Durante cuántos días permanecieron en Marruecos en esas primeras vacaciones?

25. ¿Cuándo nació la hija de Laila? 
26. ¿Cuántos años transcurren aproximadamente desde el comienzo hasta el final de la historia?

\section{Uso de la lengua}

27. Busca en un diccionario (impreso o digital) el significado de las siguientes palabras: retales (p. 7), vigas (p. 8), musulmán (p. 14), pereza (p. 39), chilaba (p. 44), henna (p. 52), mezquita (p. 59), tramitado (p. 89), comadrona (p. 115), cúmulo (p. 115).

28. Localiza en la página ocho del libro todas las palabras que encuentres pertenecientes al campo semántico de los colores.

29. Escribe un sinónimo para cada una de las siguientes palabras. Entre paréntesis figura el número de la página en la que lo encontrarás: discutían (p. 6), agotado (p. 13), regresar (p. 49), distinguir (p. 97) y alegre (p. 116).

30. Recoge un antónimo de las siguientes palabras que hallarás en las páginas indicadas: claridad (p. 15), antipáticas (p. 56), desagradables (p. 102), soportable (p. 105) y tristeza (p. 116).

31. Explica por qué se escriben con b las palabras seleccionadas: añoraba (p. 11), buscar (p. 37), blanco (p. 47), habían (p. 58) y costumbres (p. 119).

32. Especifica el motivo de la presencia de la tilde en las palabras que figuran a continuación: crepúsculo (p 19), ayudó (p. 35), tenía (p. 67), mí (p. 87), carácter (p. 104).

33. Analiza sintácticamente las siguientes oraciones:

- El avión había llegado (p. 11).

- El paseo fue muy corto (p. 21).

- Todos pedían ayuda a todos (p. 35).

- Las enfermeras eran muy simpáticas (p. 56).

- Cumplió su promesa al día siguiente (p. 109).

\section{III}

\section{Actividades de ampliación de la lectura}

\section{Creatividad}

34. ¿Qué otro título le pondrías al libro? Argumenta tu elección.

35. Inventa un final distinto para la historia.

36. Realiza un dibujo relacionado con el contenido del libro.

\section{Investigación}

37. Ubica en un mapamundi los siguientes países que aparecen citados en el libro y algunos de los cuales desempeñan un papel crucial en la historia: Marruecos, Argelia, España y Holanda.

38. Busca información en Internet sobre Marruecos y recógela en un esquema.

39. Laila y su familia eran musulmanes. Indaga acerca del Islam y del Ramadán. Sintetiza dicha información y anótala en tu cuaderno.

40. Los musulmanes están obligados a hacer varias oraciones en distintos momentos del día. Consulta la página dieciocho del libro y especifica cuántas realizaban y cuándo.

41. ¿Cuál es la lengua oficial en Marruecos? ¿Y en España?

42. La moneda de Marruecos recibe el nombre de dirham. Intenta calcular su equivalencia con respecto a la peseta y al euro, es decir, la cantidad de dirhams que equivaldrían a una peseta y a un euro.

43. La comida típica de Marruecos es el cuscús. Averigua los ingredientes necesarios para elaborar este plato y escribe la receta del mismo.

44. En la página noventa del libro se comenta la celebración de la boda de la hermana de Laila según las costumbres propias de su cultura. Comenta las diferencias con respecto a la manera de celebrar el matrimonio en nuestra cultura.

45. Busca en Internet la canción titulada Papeles mojados del grupo Chambao. Dicha canción refleja la triste situación que viven muchos/as inmigrantes, quienes arriesgan la vida al abandonar su país en unas condiciones lamentables y muy peligrosas, pues cruzan el Estrecho en pateras que en no pocas ocasiones acaban hundiéndose y provocando cuantiosas pérdidas humanas. Expresa tu opinión sobre el contenido de la misma.

\section{Reflexión, opinión y debate}

46. Como ya sabes, Laila y su familia emigran de Marruecos a España buscando unas condiciones de vida más prósperas. En la actualidad, son muchas las personas que acuden a nuestro país para lograr un puesto de trabajo que les permita sobrevivir. ¿Qué crees que podríamos hacer para que estas personas se sintiesen a gusto y se adaptasen con facili- 
dad? ¿Consideras que todos/as respetamos a los/as inmigrantes? Razona tu respuesta, coméntala con tus compañeros/as y propón soluciones para acabar con la discriminación a la que se ven sometidos algunos/as inmigrantes.

47. Lee atentamente la siguiente afirmación de Laila extraída del libro: "Mi padre quería que estudiase una carrera [...] En cambio, mi madre no lo veía así. Decía que estudiar "es perder el tiempo", porque las mujeres estamos hechas para estar en casa. Me lo repetía continuamente”. ¿Estás de acuerdo con la afirmación anterior? Argumenta tu respuesta ante tus compañeros/as y comenta algunas soluciones para acabar con la desigualdad existente entre hombres y mujeres en la sociedad.

Una vez que has realizado las actividades propuestas, habrás podido comprobar que para Laila el hecho de vivir entre dos culturas opuestas, lejos de ser un problema para ella, ha sido una experiencia muy enriquecedora que le ha brindado la posibilidad de crecer como persona. Son muchas las expresiones que así lo manifiestan: "Me siento española y privilegiada por poder conocer dos culturas diferentes, opuestas, y cada una con su magia y su encanto" (Karrouch, 2007, p. 118).

He aprendido a convivir con gente que tiene una mentalidad muy distinta de la mía. He aprendido a valorar más a las personas, a compartir sentimientos y a intercambiar opiniones.

No he perdido mi cultura ni mis raíces, sino que he ganado otra cultura y otras costumbres. Me gusta hacer un buen cuscús para comer y una tortilla de patatas para cenar. ¿Por qué no? (Karrouch, 2007, p. 119)

\section{6.- REPERCUSIÓN SOBRE COMUNIDAD EDUCATIVA.}

LA

Tras resolver las cuestiones planteadas, el alumnado no sólo valora la lectura como fuente de placer y vía de acceso al conocimiento y a la información, sino que además está preparado para entender que no hay lenguas ni culturas superiores a otras, ya que todas gozan de la misma relevancia.

De hecho, es la diversidad lingüística y cultural la que otorga riqueza a la sociedad en la que vivimos. No importa el color de la piel, ni la lengua, ni las costumbres y tradiciones típicas de cada cultura, sino que seamos ciudadanos/as responsables de una sociedad democrática en la que convivamos de forma pacífica y en la que la igualdad de género deje de ser una utopía y se convierta en una realidad.

Ahora bien, es necesario que todos/as aportemos nuestro granito de arena a la hora de construir un mundo mejor, en el que reine la paz, la tolerancia, el respeto y la justicia. Evidentemente, la puesta en práctica de actividades de este tipo repercute de una forma positiva en el clima de convivencia de la clase y del centro en general, al favorecer el respeto y la cooperación.

En el momento en que se produce el descubrimiento de la lectura como fuente de placer por parte del alumnado, podemos decir que hemos ganado un/a lector/a. "Un niño se hará lector cuando descubra que leer es un placer, un placer que le deparará buenos ratos y del que no podrá desprenderse" (Lage, 2006, p. 68). Pero no debemos olvidar que, tal y como apunta Bamberger, "el desarrollo de intereses lecturales y la formación del hábito de leer constituyen un largo proceso que empieza en el hogar paterno, se acelera sistemáticamente en la escuela y prosigue durante el resto de la vida" (1975, p. 107). "Seguir trabajando en el fomento de la lectura en todos los ámbitos, si cabe con más fuerza aún, es una necesidad para conseguir que, poco a poco, los alumnos lean. Y, con ello, se contribuirá a lograr una sociedad mejor" (Murillo et al., 2003, p. 178). Inmersos de lleno en una sociedad en la que la autoeducación continua y la autoinstrucción ininterrumpida están a la orden del día, la lectura se convierte en una herramienta crucial para potenciar el autoaprendizaje y contribuir al desarrollo de la competencia de aprender a aprender, que nos permitirá crecer como personas y mejorar nuestra formación progresivamente durante toda nuestra vida.

\section{7.- CONCLUSIONES.}

A grandes rasgos, podemos decir que, gracias a nuestra propuesta didáctica, hemos contribuido a la formación integral de nuestro alumnado. Por un lado, hemos practicado la lectura, instrumento esencial para acceder a todas las áreas del currículo, y por otro lado, hemos procurado inculcar al alumnado toda una serie de valores positivos que favorezcan su integración como ciudadanos/as responsables en el seno de la sociedad democrática en que vivimos, cuyas 
características más acuciantes son la diversidad lingüística y cultural, muestra palpable de riqueza. Muy acertadas son las palabras que profiere Mata en relación con el apasionante trabajo de educar. A su juicio, educar "no significa acomodar a los alumnos al presente sino prepararlos para el mundo por venir, para el mejor y más justo de los mundos concebibles. La educación es el umbral que da paso al ejercicio de la ciudadanía" (2004, p. 123). En esta tarea, la lectura, como transmisora del saber de la humanidad, desempeña un papel primordial. Culminaremos esta reflexión acerca de la importancia de la lectura con una cita sobre la misma: "Leer es abrir una puerta a huéspedes desconocidos, a pensamientos extraños que llegan sin previo aviso, sin señales reconocibles. Muchos de ellos apenas descansarán unas horas en nuestra conciencia, pero otros habitarán en nuestra memoria para siempre" (Mata, 2004, p. 29).

\section{8.- REFERENCIAS BIBLIOGRÁFICAS.}

Bamberger, R. (1975). La promoción de la lectura. Barcelona: Ediciones de Promoción Cultural.

Casas, L. \& Centellas, J. (1989). Yo leo. Una experiencia de biblioteca de aula en el Ciclo Medio. Barcelona: Pirene.

Castán, G. (2002). Las bibliotecas escolares (soñar, pensar, hacer). Sevilla: Díada.

Decreto 231/2007 de 31 de julio, por el que se establece la ordenación y las enseñanzas correspondientes a la Educación Secundaria Obligatoria en Andalucía (BOJA n ${ }^{\circ}$ 156, de 8 de agosto de 2007).
Gardner, J. \& Myers, L. (2005). La lectura es divertida. Diez métodos para cultivar el hábito de la lectura en los niños. Alcalá de Guadaíra: MAD.

Karrouch, L. (2007). Laila. Barcelona: Planeta \& Oxford.

Lage, J. J. (2006). Animar a leer desde la biblioteca. Madrid: CCS.

Ley Orgánica 2/2006, de 3 de mayo, de Educación (BOE n⿳0 106, de 4 de mayo).

Martín, D. (2005). Programa de animación a la lectura y escritura. Málaga: Aljibe.

Mata, J. (2004). Como mirar a la luna. Confesiones a una maestra sobre la formación del lector. Barcelona: Graó.

Murillo, F. J., Lucio-Villegas, M., Asensio, M., Brioso, M. J., Cuesta, J. C., Rodríguez, M. \& Sánchez A. (2003). Los hábitos lectores de los adolescentes españoles. Madrid: Ministerio de Educación, Cultura y Deporte.

II Plan Integral para la Inmigración en Andalucía. Consejería de Gobernación de la Junta de Andalucía (BOJA no 105, de 2 de junio de 2006).

Real Decreto 1631/2006 de 29 de diciembre, por el que se establecen las enseñanzas mínimas correspondientes a la Educación Secundaria Obligatoria (BOE $\mathrm{n}^{\circ} 5$, de 5 de enero de 2007).

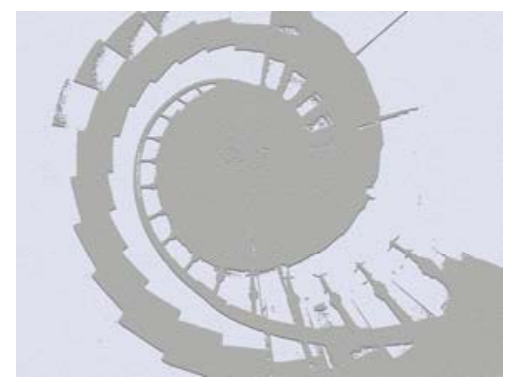

